\title{
Azido\{1-[2-(dimethylamino)ethyliminomethyl]- naphthalenolato\}copper(II). Erratum
}

\author{
Qi-Yong Zhu, Yi-Jun Wei* and Feng-Wu Wang \\ Department of Chemistry, Huainan Normal College, Huainan 232001, People's Republic of China. ${ }^{*}$ Correspondence \\ e-mail: huainanweiyijun@163.com
}

Received 3 February 2016

Accepted 3 February 2016

In the paper by Zhu et al. [Acta Cryst. (2006), E62, m983-m985], the metal atom was reported incorrectly.

Edited by W. T. A. Harrison, University of Aberdeen, Scotland

The authors of Zhu et al. (2006) have indicated that the metal atom in the reported complex was assigned incorrectly. The paper is therefore withdrawn from the published literature and associated databases.

\section{References}

Zhu, Q.-Y., Wei, Y.-J. \& Wang, F.-W. (2006). Acta Cryst. E62, m983m985. 